\title{
A MuSIC-al way to identify drug pairs
}

Combinations of drugs could provide improved benefits for treating several disorders, including HIV, hepatitis C virus and bacterial infections, as well as malaria and cancer, but the vast number of potential drug combinations makes it difficult and/or costly to systematically identify these. Now, writing in Nature Biotechnology, Tan and colleagues describe a new method - dubbed MuSIC (multiplex screening for interacting compounds) - that identified synergistic drug pairs and new targets for the potential treatment of HIV and could be widely applicable to screens in other therapeutic areas.

The authors selected 1,000 test compounds from two commercially available drug libraries and from the US National Institutes of Health's clinical compound collection. The design of the MuSIC screen was based on a heuristic algorithm to cover all the possible pairwise combinations (that is, $~ 500,000$ drug pairs) of these compounds. The initial assay used forty-five 384-well plates, each containing a pool of 10 compounds per well (this was $<3 \%$ of the number of wells needed in a standard pairwise screen). The screen measured HIV infectivity and was designed to detect effects on both the early and late stages of the HIV life cycle.

An initial primary screen identified 288 drug pools based on low infection rate and low cytotoxicity, which were then disseminated into 12,904 drug pairs. These drug pairs were subjected to a secondary screen (another heuristic algorithm was used to design the layout of the plates), which identified 104 drug pairs that reduced the HIV infection rate by $\geq 50 \%$. Two established models were then used to measure synergy between the identified drug pairs: the Bliss independence model and higher single activity model. Forty-one drug pairs were synergistic using both models. The top-ranked pair included the glucocorticoid betamethasone (which is known to reduce HIV transcription) and the antiprotozoal drug nitazoxanide (whose anti-HIV activity has not been previously reported). Other high-ranked compounds included glucocorticoids, non-steroidal antiinflammatory drugs (NSAIDs) and anticholinergic drugs.

Further work demonstrated that nitazoxanide and glucocorticoids such as betamethasone target different steps of the HIV life cycle. Nitazoxanide acted at or near the HIV reverse transcription step, whereas glucocorticoids inhibited a step after reverse transcription.

Next, the authors used computational analysis of the results of the secondary screen to derive a drug-drug interaction network that ranked compounds according to antiHIV activity and synergy between drugs. Top-ranked drugs included glucocorticoids, NSAIDs, muscarinic cholinergic receptor antagonists and quinolone. Notably, the network was enriched for anti-inflammatory drugs. Moreover, the interaction network identified drugs inhibiting viral assembly and release that were not previously known to have anti-HIV effects, such as calcium channel blockers, cytochrome P450 inhibitors and $\alpha$-adrenergic receptor antagonists.

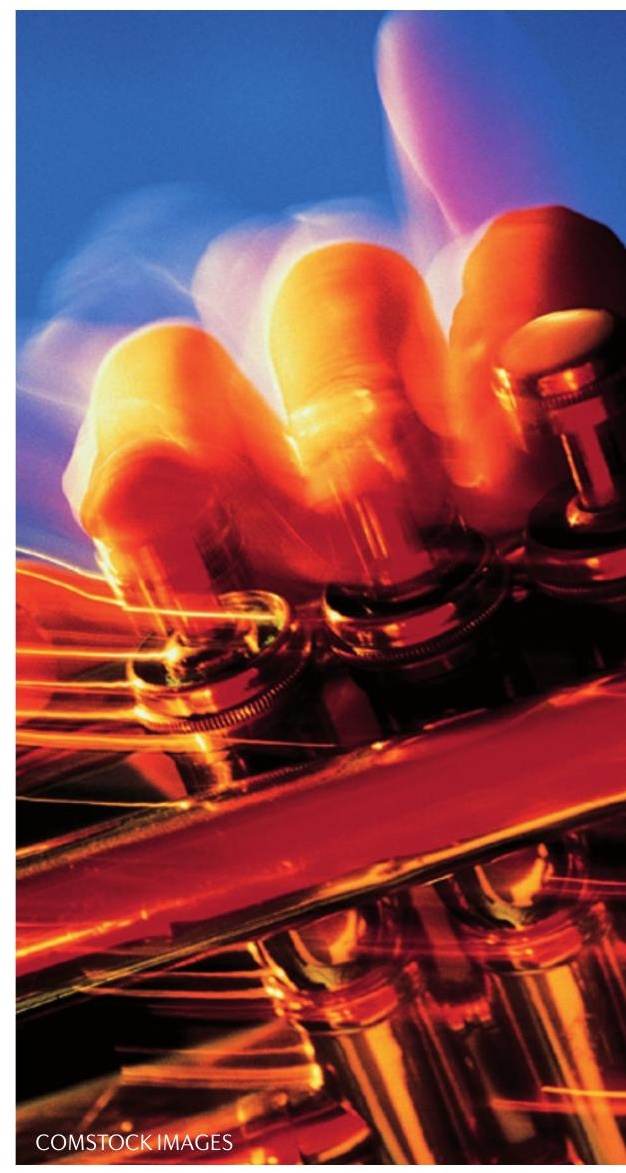

So the MuSIC assay identified drug pairs that could have potential for the treatment of HIV. The authors note that the assay could be used for a variety of disease-relevant screens to enable the efficient repositioning of drugs and drug pairs.

\section{Charlotte Harrison}

ORIGINAL RESEARCH PAPER Tan, X. et al. Systematic identification of synergistic drug pairs targeting HIV. Nature Biotech. 14 Oct 2012 (doi:10.1038/nbt.2391) 